\title{
Intraoperative fluorescence imaging during complex urologic pelvic surgery
}

\begin{abstract}
Extirpative therapy remains an important treatment for the management of invasive pelvic cancers. Radical cystectomy or anterior pelvic exenteration with urinary diversion has the potential for postoperative morbidity and mortality. Several critical components of these procedures such as delicate anastomoses can be affected by potential complications related to ischemia and are subject to the limitations of visualization with only white light in the operating room. Indocyanine green (ICG) is a fluorescent dye that has several applications in Urology. We examine the implementation of ICG within pelvic extirpative therapies as well as review the many uses of ICG within Urologic procedures.
\end{abstract}

Keywords: ICG, cystectomy, exenteration
Volume 8 Issue 3 - 2020

\author{
Srinath Kotamarti, Ervin Teper,Ariel \\ Schulman \\ Maimonides Medical Center, Brooklyn NY
}

Correspondence: Srinath Kotamarti, Division of Urology, Department of Surgery, Maimonides Medical Center 4802 10th Avenue, Brooklyn NY II219, 718-283-7153, USA, Email srikotaarti@gmail.com

Received: May 18, 2020 | Published: June 09, 2020

\author{
Abbreviations: ICG, indocyanine green; RC, radical \\ cystectomy
}

\section{Introduction}

Pelvic extirpative therapy remains a mainstay in the treatment paradigm for the management of invasive genitourinary, gynecologic and colorectal cancers. With regards to Urology, radical cystectomy (RC) or anterior pelvic exenteration with urinary diversion is the gold standard for treatment for MIBC with perioperative complication rate of up to $70 \% .^{1}$ Indeed, these surgeries are associated with high rates of postoperative morbidity and mortality. ${ }^{2}$ The balance to be struck between maintaining both maximal oncologic outcome as well as lowest morbidity with these procedures is crucial. In invasive urologic malignancies involving the bladder and urethra, often urinary diversion is required, exposing the patient to further potential complications regarding the incorporation of bowel into the tract. Performing these procedures involves important critical components including the stoma formation, bowel anastomosis, uretero-intestinal anastomosis, as well as any indicated flap necessary for defect closure post resection. All these steps may be subject to potential complications related to leakage of fecal contents, sepsis, wound infections, abscesses, anastomotic stenosis, hemorrhage, obstruction, wound dehiscence and flap necrosis, among others., ${ }^{2,3}$ In the settings of post-radiation or immunosuppression, these complications occur at even higher rates. Often these aforementioned complications are related to ischemia. The procedures rely on the expertise of the surgeons to identify viable tissues and delicately create viable anastomoses. However, current standard operative practices leave the surgeon with only gross vision and white light to identify adequately perfused tissues. In the case of uretero-enteric anastomoses, for example, stricture rates as high as $2.6 \%$ are deemed acceptable. ${ }^{4}$ In addition, certain complications including bowel leak are even associated increased risk of perioperative mortality. ${ }^{5}$ If such adverse events can be avoided, the patient has the opportunity to be spared extensive morbidity. As such, the need exists to develop intraoperative tools to further improve outcomes.

\section{Indocyanine green and its administration}

Indocyanine green (ICG) is a cyanine dye that fluoresces at $830 \mathrm{~nm}$ when exposed to incident infrared light of wavelength $780 \mathrm{~nm} .{ }^{6}$ The dye is primarily retained in the intravascular space where it binds to plasma proteins. ${ }^{6}$ Administered intravenously and used in conjunction with a camera and software-imposed pseudo-color, it may be used to identify vessel perfusion and differentiate tissue density. ${ }^{6}$ The fluorescence emitted by ICG may be identified utilizing specific scopes and cameras. Thus, further information may be attained regarding anatomy, perfusion, or lymphatic drainage. ${ }^{7}$ Standard dosing up to 5 $\mathrm{mg} / \mathrm{kg}$ intravenously is considered non-toxic with iodine allergy as the only major contraindication. It features a favorable adverse effect profile, with a low complication rate of $0.34 \%$ of nausea, vomiting and rarely shock. ${ }^{8}$ Cleared via the liver, half-life of the drug is 3-4 minutes with clearance from the system within 10-20 min, allowing for multiple administrations. ${ }^{9}$

ICG may be utilized in both open as well as minimally invasive techniques. Originally developed in 2005 by Novadaq (Mississauga, Ontario) as the Spy Imaging System (Figure 1), it was further improved to incorporate endoscopic versions called the Spy Scope and subsequently rebranded as the Pinpoint system (Figure 2). ${ }^{6}$ Generally, the system is comprised of a large camera head on a boom, which is arrayed above the surgical field as well as software to quantify tissue perfusion. Fluorescence imagery is portrayed with poorly perfused tissue appearing almost black in appearance. The Pinpoint system incorporates both white light as well as near-infrared (NIR) fluorescence excitation into a single camera head. Proprietary software displays the near infrared image as an overlay over the white light image in real-time as neon green highlighting, allowing the surgery team access to both throughout the procedure to provide realtime guidance. ${ }^{6}$ Implemented in robotic surgery using the da Vinci ${ }^{\circledR}$ robot (Intuitive Surgical Systems, Sunnyvale CA USA), it is known as the Firefly ${ }^{\circledR}$ system. ${ }^{10}$ For open procedures, a handheld version exists as well - the SPY-PHI by Novadaq (Figure 3$)^{6}$ 


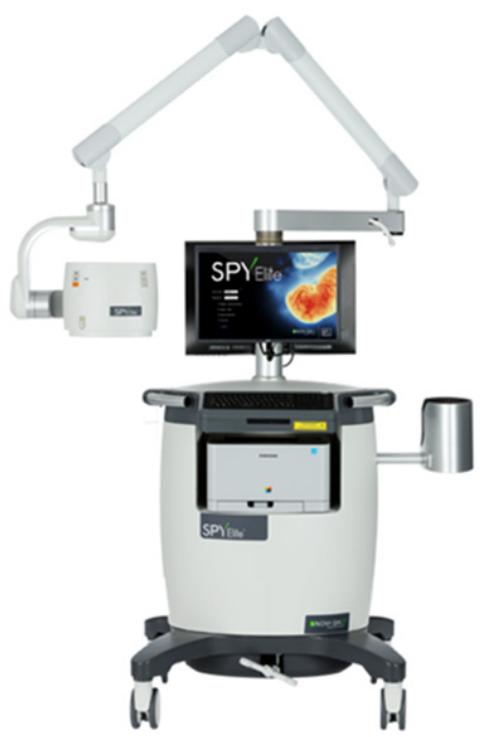

Figure I SPY Imaging system by Novadaq (www.novadaq.com).

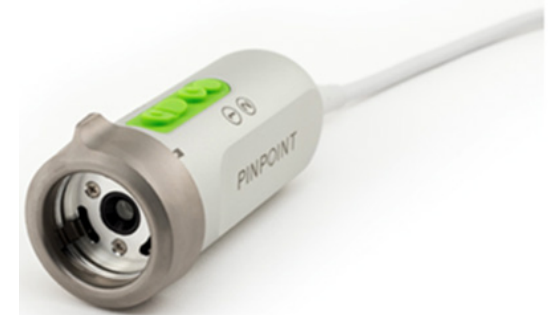

Figure $\mathbf{2}$ The endoscopic pinpoint system by novadaq (www.novadaq.com).

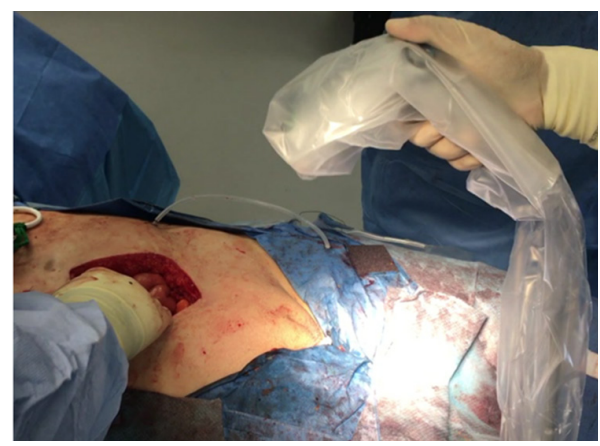

Figure 3 The SPY-PHI handled system for fluorescence imaging.

To administer ICG, the solution is first prepared via combining and mixing $25 \mathrm{mg}$ of the dye in distilled water. ${ }^{10}$ Just prior to creation of the uretero-enteric anastomosis or other step in question, $10 \mathrm{~mL}$ of solution is injected intravenously. ${ }^{10}$ Subsequently, the anatomic areas in question are examined via the NIR fluorescence system. In our institution for complex open pelvic extirpative procedures we have used the handheld SPY PHI system for this purpose. The time between ICG injection and visualization of fluorescence is noted. Examination of both the arterial supply as well as the tissues during both the arterial and perfusion phases of ICG is undertaken. It has previously been reported that it takes approximately 30 seconds after injection to assess arterial flow and around 5 minutes to appropriately be able to assess perfusion. ${ }^{10}$ Once fluorescence was visualized, it should give a clear demarcation line between well-perfused and non-perfused colon leading to a correct resection line. For difficult to discern situations, the surgeon is then able to toggle between white light and NIR prior to proceeding. ${ }^{10}$

\section{Applications of ICG within cystectomy}

Within radical cystectomy there are several potential uses for ICG to aid the surgeon in peri-operative planning. Several studies have examined the uretero-intestinal anastomosis related to urinary diversion. Ahmadi et al. ${ }^{10}$ in their cohort of 179 patients undergoing robotic cystectomy with urinary diversion found that patients receiving ICG had greater length of ureter being excised during uretero-enteric anastomosis with no uretero-enteric strictures compared to a $10.6 \%$ rate of stricture per patient in those who did not receive ICG. ${ }^{10}$ Figure 4 demonstrates ICG utilization for the uretero-enteric anastomosis during an open approach, showing slightly decreased perfusion without complete nonviability of the anastomosis. Bowel anastomosis provides another opportunity for ICG implementation. While not described in Urologic reports, ICG has been utilized extensively in colorectal surgery in terms of maximizing performance of bowel anastomosis. Ris et al. ${ }^{11}$ found ICG angiography was associated with change in site of bowel division in $5.8 \%$ of patients with no subsequent leaks compared to a $2.4 \%$ overall leak rate in their Phase II cohort of 504 men undergoing elective colorectal surgery with bowel anastomosis. ${ }^{11}$ A potentially nonviable bowel anastomosis segment is appreciated in Figure 5.
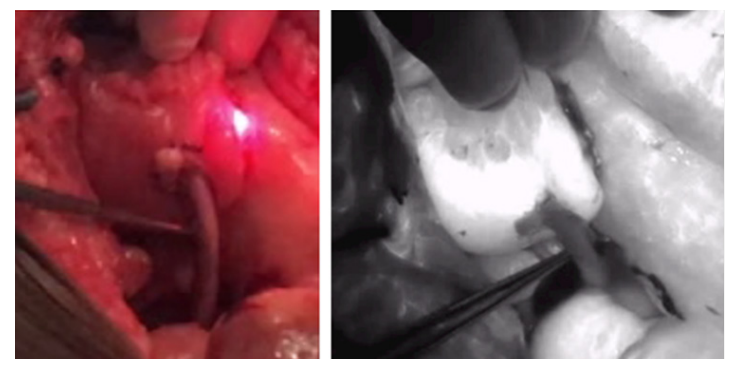

Figure 4 ICG utilization (right) versus white light (left) during uretero-enteric anastomosis as part of anterior pelvic exenteration demonstrating at least partial viability of the ureteric anastomotic segment (does not appear pure black in color).
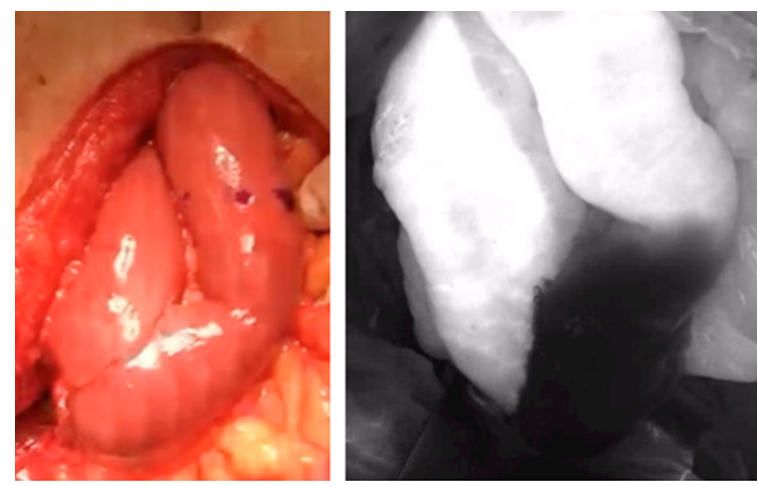

Figure $\mathbf{5}$ White light (left) and NIR imaging (right) of open bowel anastomosis during cystectomy demostrating non-viable segment of the bowel anastomosis.

In addition, the stoma site itself can be subject to complications such as stomal stenosis related to undue tension on the conduit pedicle as the stoma is brought through the skin, leading to pressure on the vasculature. Typically, a pink appearance is considered acceptable for the stomal opening and is generally deemed sufficient to indicate healthy tissue, however, ICG provides the potential to confirm viability (Figure 6). While no current reports exist on this aspect, 
theoretically ICG utilization to assess stomal vascularization could have a contributing hand to decrease need for stomal revisions. ${ }^{12}$ Lastly, in certain complex cases of aggressive resection for pelvic disease, vascularized flaps may be required to provide tissue coverage for large defects in the pelvis or perineum. Lack of adequate tissue for primary closure or prior radiation provides challenges to the operating team. To prevent poor or non-healing wounds and provide adequate support which can be obtained via plastic surgery placement of local or distant flaps with or without skin like the vertical rectus abdominis muscle flap (VRAM) or free flaps like the latissimus dorsi flap. ${ }^{13}$ In these cases, identifying and incorporating wellperfused flaps are crucial to ensure optimal post-operative outcome. As appreciated in Figure 7, ICG can also be implemented to assess perfusion and viability of the flap prior to closure. Thus, there are multiple opportunities during complex pelvic extirpative procedures including radical cystectomy for ICG to assess tissue perfusion and reduce tissue and patient complications.

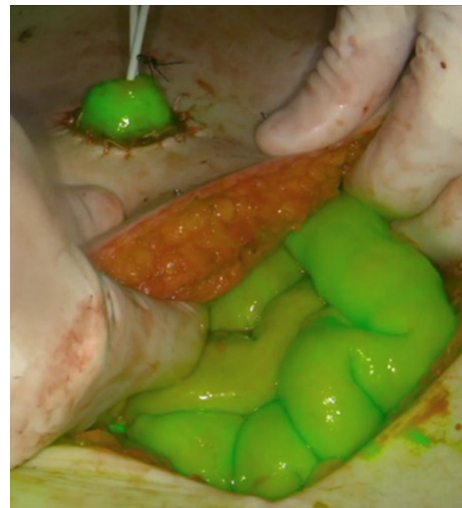

Figure 6 ICG utilization confirming vascular viability of the ileal conduit stoma during pelvic exenteration.
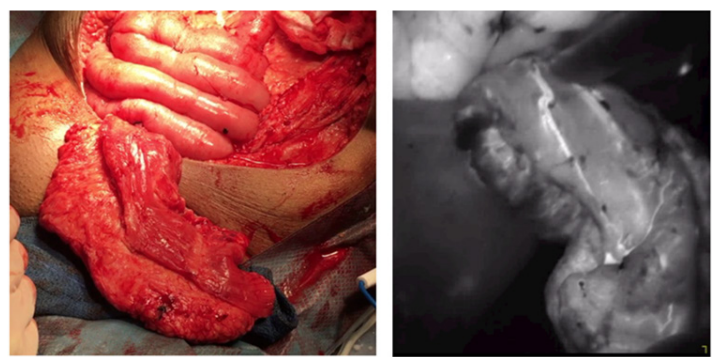

Figure 7 Fluorescent imaging depicting adequate vascularity and blood flow to the vertical rectus abdominis muscle (VRAM) flap to be utilized for wound coverage during pelvic exenteration. White light imaging appreciated on the left.

\section{Other utilizations of ICG within urology}

While already established in other fields like breast and plastic surgery, ICG has been implemented with success in other aspects of urologic surgery, as well, including robotic procedures. ${ }^{14,15}$ The dye has been used as an angiographic aid with regards to partial nephrectomy to identify the tumor arterial blood supply. In this setting, ICG theoretically allows for selective clamping and limiting ischemia time. ${ }^{16}$ Even after resection of the tumor, ICG may be utilized to assess the viability of residual renal parenchyma. ${ }^{16}$ Similarly, ICG angiography may aid in identification of ureteral obstruction related to scarring. ${ }^{17}$ In prostate and penile cancers, ICG bound to a carrier prior to injection such as $99 \mathrm{mTechnetium} \mathrm{has} \mathrm{been} \mathrm{used} \mathrm{previously}$ to map and predict patterns of lymphatic drainage and aid in more directed and higher yield node dissections. ${ }^{18,19}$ Furthermore, there have been efforts to implement ICG as a means of identifying the benchmark artery during robotic-assisted radical prostatectomy to improve preservation of the neurovascular bundle during nervesparing approaches..$^{20}$ ICG has also been considered for intraluminal administration, as it has been suggested that ureteral identification as well as ureteral stricture location may be optimized with intra-ureteral ICG instillation for robotic upper tract reconstructive procedures. ${ }^{17}$

\section{Future of ICG}

As with numerous other types of procedures, both Urologic and non-Urologic, radical cystectomy and other pelvic extirpative procedures provide numerous potential uses for ICG to reduce surgical toxicity. Further development is ongoing to potentially produce enhanced fluorescence-based tools to overcome the limiting effect of tissue attenuation. For example, NIR fluorophores for targetspecific staining are currently under development for use in prostate and rectal cancers. ${ }^{21,22}$ Furthermore, the combination of multiple fluorophores could provide the potential to possibly provide even more detail on anatomical structures peri-operatively. In addition, implementation of ICG into a standardized peri-operative protocol regimen such as an enhanced recovery after surgery (ERAS) protocol provides an attractive means to enhance peri-operative care. ERAS protocol already exists for radical cystectomy, with proven outcomes including significantly shorter hospital stays with shorter time to bowel function than those undergoing standard protocol. ${ }^{23}$ In colorectal surgery, feasibility and safety of ICG implementation within ERAS has been explored with demonstration of significant reduction in anastomotic leak while maintaining similar operative times. ${ }^{7}$ Thus, ICG has a potential role to play within expedited perioperative protocols for Urologic pelvic extirpative surgery. Not only is fluorescence technology safe and easy to use, but it serves as a means to maximize oncologic outcome while reducing comorbidity in the patient requiring pelvic surgical resection.

\section{Acknowledgments}

None.

\section{Conflicts of interest}

The author declares there is no conflict of interest.

\section{Funding}

None.

\section{References}

1. Chang SS, Bochner BH, Chou R, et al. Treatment of Non- Metastatic Muscle-Invasive Bladder Cancer: AUA/ASCO/ASTRO/SUO Guideline. J Urol. 2017;198(3):552-559.

2. Tortorella L, Casarin J, Mara KC, et al. Prediction of short-term surgical complications in women undergoing pelvic exenteration for gynecologic malignancies. Gynecol Oncol. 2019;152(1):151-156.

3. Hautmann RE, de Petriconi RC, Volkmer BG. Lessons learned from 1000 neobladders: the 90-day complication rate. J Urol. 2010;184:990-994.

4. Shah SH, Moyassaghi K, Skinner D, et al. Ureteroenteric Strictures After Open Radical Cystectomy and Urinary Diversion: The University of Southern California Experience. Urology. 2015;86(1):87-91. 
5. Mizrahi I, Abu-Gazala M, Rickles AS, et al. Indocyanine green fluorescence angiography during low anterior resection for low rectal cancer: results of a comparative cohort study. Tech Coloproctol. 2018;22(7):535-540.

6. Lewis JL, Sherwinter DA. The Pinpoint system. Fluorescence Imaging for Surgeons: Concepts and Applications. Springer International Publishing Switzerland. 2015

7. Brescia A, Pezzatini M, Romeo G, et al. Indocyanine green fluorescence angiography: a new ERAS item. Updates Surg. 2018;80(4):427-432.

8. Obana A, Miki T, Hayashi K. Survey of complications of indocyanine green angiography in Japan. Am J Ophthalmol. 1994 Dec;118(6):749-53.

9. van der Poel HG, Grivas N, van Leeuwen F. Comprehensive Assessment of Indocyanine Green Usage: One Tracer, Multiple Urologic Applications. Eur Urol Focus. 2018;4(5):665-668.

10. Ahmadi N, Ashrafi AN, Hartman N, et al. Use of indocyanine green to minimize uretero-enteric strictures after robotic radical cystectomy. BJU Int. 2019124(2):302-307.

11. Ris F, Liot E, Buchs NC, et al. Multicentre phase II trial of near-infrared imaging in elective colorectal surgery. Br J Surg. 2018;1059(10):13591367.

12. Richardson JR Jr, Linton PC, Leadbetter GW Jr. A new concept in the treatment of stomal stenosis. J Urol. 1972;108(1):159-161.

13. Abdou A, Bruns H, Troja A, et al. Plastic surgery of extended defects after exenteration of the pelvis. Zentralbl Chir. 2015;140(2):214-218.

14. Bates AS, Patel VR. Applications of Indocyanine green in robotic urology. J Robot Surg. 2016;10(4):357-359.

15. Bjurlin MA, Gan M, McClintock TR, et al. Near-infrared Fluorescence imaging: Emerging applications in Robotic Upper Urinary Tract Surgery. Eur Urol. 2014;65:793-801.
16. McClintock TR, Bjurlin MA, Wysock JS, et al. Can selective arterial clamping with fluorescence imaging preserve kidney function during robotic partial nephrectomy? Urology. 2014;84:327-332

17. Lee Z, Moore B, Giusto L, et al. Use of indocyanine green during robotassisted ureteral reconstructions. Eur Urol. 2015;67:291-298.

18. Wit EM, Acar C, Grivas N, et al. Sentinel node procedure in prostate cancer: a systematic review to assess diagnostic accuracy. Eur Urol. 2017;71:596-605.

19. Sávio LF, Barboza MP, Alameddine M, et al. Combined partial penectomy with bilateral robotic inguinal lymphadenectomy using nearinfrared fluorescence guidance. Urology. 2018;113:251.

20. Mangano MS, DeGobbi A, Benjamin F, et al. Robotic-assisted nervesparing radical prostatectomy ursing near-infrared fluorescence technology and indocyanine green: initial experience. Urologia. 2018;85(1):29-31.

21. Neuman BP, Eifler JB, Castanares M, et al. Real-time, near-infrared fluorescence imaging with an optimized dye/light source/camera combination for surgical guidance of prostate cancer. Clin Cancer Res. 2015;21:771-780.

22. Rijpkema M, Bos DL, Cornelissen AS, et al. Optimization of duallabeled antibodies for targeted intraoperative imaging of tumors. Mol Imaging. 2015;14:348-355.

23. Frees SK, Aning J, Black $\mathrm{P}$, et al. A prospective randomized pilot study evaluating an ERAS protocol versus a standard protocol for patients treated with radical cystectomy and urinary diversion for bladder cancer World J Urol. 2018;36(2):215-220. 